\title{
Up-regulated expression of stathmin may be associated with hepatocarcinogenesis
}

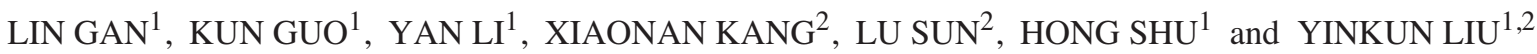 \\ ${ }^{1}$ Liver Cancer Institute, Zhongshan Hospital, ${ }^{2}$ Institute of Biomedical Science, \\ Fudan University, Shanghai 200032, P.R. China
}

Received November 10, 2009; Accepted December 23, 2009

DOI: 10.3892/or_00000730

\begin{abstract}
Stathmin is a microtubule-destabilizing protein ubiquitously expressed in vertebrates and overexpressed in a variety of human malignancies. Down-regulation of its expression will contribute to optimize therapeutic outcomes in the treatment of these malignancies. This research aimed to demonstrate effects of stathmin expression silencing on hepatocellular carcinoma (HCC) cell proliferation, apoptosis, cell adhesion and motility behavior in vitro and further reveal significance of stathmin expression in tissues associated with hepatocarcinogenesis. The expression of stathmin in normal liver, hepatitis, cirrhosis and HCC tissues was detected by immunohistochemistry analysis (IHC), stathmin expression was inhibited in an HCC cell line-HCCLM3 with high metastatic potential by small interfering RNAs (siRNAs). After transfection with siRNAs, HCCLM3 cells proliferation was detected by CCK-8 (cell count kit), cell apoptosis was analyzed by FACS, cell adhesion was investigated by cell adhesion assay and motility ability was demonstrated by in vitro migration and invasion assays. Stathmin expression was up-regulated in HCC tissues, especially in metastatic HCC tissues, compared with normal liver, hepatitis and hepatic cirrhosis tissues. Expression of stathmin in HCCLM3 cells was efficiently inhibited by specific siRNAs. Silencing of stathmin expression obviously suppressed HCCLM3 cell proliferation and markedly induced cell apoptosis. Moreover, defect of stathmin expression in HCCLM3 cell inhibited cell adhesion, restrained cell migration and repressed invasion. Stathmin expression correlates with hepatocarcinogenesis and tumor progression. This molecule may be a promising therapeutic target in patients with hepatocellular carcinoma.
\end{abstract}

Correspondence to: Dr Yinkun Liu, Liver Cancer Institute, Zhongshan Hospital, Fudan University, Shanghai 200032, P.R. China E-mail: liu.yinkun@zs-hospital.sh.cn

Key words: hepatocellular carcinoma, stathmin, RNA interference, carcinogenesis

\section{Introduction}

Hepatocellular carcinoma (HCC) is the fifth most common cancers in the world and the third most common cause of cancer mortality. It was estimated that there are nearly 700,000 new cases annually and almost as many deaths, and $82 \%$ of cases occur in developing countries (1). It is reported that more than $50 \%$ HCC cases occur in China (2). Globally, $\mathrm{HBV}$ infection is the most frequent important cause of HCC, and over $90 \%$ of HCC cases develop against a background of chronic liver injury and a high prevalence of Hepatitis B or C (3). Furthermore, it is also well-known that fibrosis and cirrhosis are the underlying steps in hepatocarcinogenesis. The great majority, about $70-90 \%$ of HBV-related HCC develop in patients with fibrosis and cirrhosis. As shown by epidemiology data, hepatocarcinogenesis is linked tightly to the development of chronic HBV infection, and associated with the evolution of fibrosis and cirrhosis.

Stathmin is also called Op18 (oncoprotein 18), metablasin, p18 or p19, which is a ubiquitously expressed $19-\mathrm{kDa}$ cytosolic phosphoprotein that can integrate various cellular regulatory signals. It is encoded by the human STMN1 gene located at chromosome $1 \mathrm{p} 36.1$ which is related to high frequency of allelic loss in HCC (4). HCC exhibits frequent chromosomal losses in 1p, 4q, 16q and 17p (5-7), especially in 1p36, which include 1p36.1 (8), 1p36.13-p36.23 $(9,10)$ and 1p36.2-p36.3 $(11,12)$. It suggests the presence of putative tumor suppressor genes in these regions. Usually the highest levels of stathmin are found in the brain, peripheral nervous system and embryonic tissues $(13,14)$, while the expression of stathmin can hardly be detected in adult liver tissue. Recently, more and more experiments have shown that stathmin is overexpressed in many human cancers, such as sarcoma, breast cancer, prostate cancer, and leukemia, which suggest that stathmin plays a crucial role in tumorigenesis and may be a key anti-oncogene in tumors $(15,16)$.

As an important microtubule-destablizing protein, stathmin has already been found to be involved in regulations of cell proliferation, apoptosis, differentiation and motility. In this study, we evaluated stathmin expression patterns in various liver tissues including normal liver, HBV hepatitis, cirrhosis, HCC tissues and reveal the roles of this protein in the process of hepatocarcinogenesis. Furthermore, the effects of stathmin expression silencing on HCC cell proliferation, 
apoptosis, adhesion and motility abilities in vitro, suggested the possibility of stathmin as a potential target in hepatocellular carcinoma therapy.

\section{Materials and methods}

Tissue microarray (TMA) and immunohistochemistry (IHC). All samples were obtained from the Department of Hepatobiliary Surgery, First Affiliated Hospital of Guangxi Medical University (Nanning, China). Access to human tissues complied with the laws of China and the guidelines of the Ethics Committee. Data do not contain any information that may lead to the identification of the patients. Matched pairs of primary HCC samples and adjacent liver tissues (each 48 cases); normal liver, hepatitis, cirrhosis and HCC tissue (24 cases, respectively); primary and metastasis HCC (each 48 case) were used for the construction of TMA (Shanghai Biochip Co., Ltd., Shanghai, China) as previously described (17). All samples from patients were reviewed histologically by $H \& E$ staining, and representative areas with small round lymphocyte infiltrate were premarked in the paraffin blocks, away from necrotic and hemorrhagic materials.

The process of IHC was according to the protocol of antistathmin antibody (ABCam, UK). Briefly, paraffin sections were first deparaffinized and then hydrated. After microwave antigen retrieval, as required, endogenous peroxidase activity was blocked with incubation of the slides in $0.3 \% \mathrm{H}_{2} \mathrm{O}_{2}$, and non-specific binding sites were blocked with $10 \%$ goat serum. After serial incubation with primary antibodies (1:1000), and secondary antibody, the sections were developed in diaminobenzidine solution under a microscope and counterstained with hematoxylin. Negative control slides omitting the primary antibodies were included in all assays.

Cell culture. A stepwise metastatic human hepatocellular carcinoma cell lines which were established by our Liver Cancer Institute: MHCC97L, MHCC97H, HCCLM3 and HCCLM6 were grown in DMEM medium (Gibco, NY, USA) supplemental with $10 \%$ fetal bovine serum (FBS) (Gibco). Another HCC cell line-Hep3B, integrated with HBV, were also used in the study, which were maintained in RPMI-1640 medium (Gibco) with 10\% FBS.

Western blot analysis. Total protein $(25 \mu \mathrm{g})$ extracted in SDS-lysis buffer were run on a $10 \%$ SDS polyacrylamide gel and electrophoretically transferred to PVDF membranes. Strips of the membranes were blocked for $1 \mathrm{~h}$ at room temperature with blocking buffer (1X TBS, $0.05 \%$ Tween-20 with $5 \%$ non-fat dry milk), incubated overnight at $4{ }^{\circ} \mathrm{C}$ with antistathmin (ABCam, 1:10000) or anti-GAPDH (Kangchen Biotech, China, 1:5000). They were incubated for $1 \mathrm{~h}$ at room temperature with HRP-conjugated secondary antibody (Amersham, UK, 1:5000). Peroxidase activity on the PVDF membrane was visualized on X-ray film by means of the ECL Western blot detection system. Bands on films were scanned and quantified using densitometric scanning of the blots and normalized against GAPDH.

$R N A$ extraction and $R T-P C R$. The total RNA was extracted by TRIzol (Invitrogen, USA). Total RNA was retrotranscripted using MMLV reverse transcriptase and random primers
(Promega, WI) including $2 \mu \mathrm{g}$ RNA; $1 \mu \mathrm{g}$ of the obtained cDNA was then amplified using primers for the human stathmin and GAPDH sequence. The sequence of primers was: stathmin, 5'-tagaatcttgagattctctctc-3' (sense), 5'-caggtc agctgttacttt-3' (antisense); GAPDH: 5'-ccatgttcgtcatgggtgtgaa cca-3' (sense), 5'-gccagtagaggcagggatgatgttc-3' (antisense). PCR was performed with initial heating at $94^{\circ} \mathrm{C}$ for $5 \mathrm{~min}$, followed by 30 cycle of $94^{\circ} \mathrm{C}$ for $30 \mathrm{sec}, 52^{\circ} \mathrm{C}$ for $30 \mathrm{ssec}$, $72^{\circ} \mathrm{C} 30 \mathrm{sec}$, and finally $72^{\circ} \mathrm{C}$ for $10 \mathrm{~min}$. PCR products were separated by $2 \%$ agarose gel electrophoresis, then scanned and analyzed by VDS imagemaster system (Pharmacia).

RNA interference. Stathmin siRNA duplex (STMN1 sense: 5'-GAAACGAGAGCACGAGAAAtt-3'; antisense: 5'-UUUC UCGUGCUCUCGUUUCtt-3', STMN2 sense: 5'-GCACGA GAAAGAAGUGCUUtt-3'; antisense: 5'-AAGCACUUCUU UCUCGUGCtt-3'; STMN3 sense: 5'-GAGAACCGAGAGG CACAAAtt-3'; antisense: 5'-UUUGUGCCUCUCGGUUCU Ctt-3') were designed and constructed from Jikai Corp. Cells $\left(2 \times 10^{6}\right)$ with $60 \%$ confluency in 6-well culture plates were transfected with a final concentration $(80 \mathrm{nM})$ of stathmin siRNA using Lipofectamine $2000^{\mathrm{TM}}$ RNAi MAX transfection reagent (Invitrogen) for $48 \mathrm{~h}$ to detect mRNA and protein level in the presence of control or stathmin siRNA according to the manufacture instruction (RNAi group). Cells which were transfected with non-specific siRNA (Mock group) or non-treated HCCLM3 (control group) were parallelly demonstrated.

Cell proliferation assay. To determine the effect of stathmin suppression on HCCLM3 cell proliferation, we used a tetrazolium reagent, 2-(4-indophenyl)-3-(4-nitrophenyl)-5(2,4-disulphophenyl)-2H-tetrazolium monosodium salt (CCK8, Cell Counting kit, Dojindo, Kumamoto, Japan). HCCLM3 ( $1 \times 10^{3}$ cells/well) were seeded in 96-well culture plates in their basal media supplemented with $10 \%$ FBS, transfected with stathmin siRNA (RNAi group) or non-specific siRNA (Mock group) for $48 \mathrm{~h}$, while non-treated HCCLM3 cells as control group. Each group was seeded in at least 3 wells, respectively. The cells were then incubated with CCK8 reagent for $1 \mathrm{~h}$ at $37^{\circ} \mathrm{C}$. The staining intensity in the medium was measured by determining the absorbance at $450 \mathrm{~nm}$.

Analysis of apoptotic cells by flow cytometry. The assay process was according to the protocol of FITC Annexin V Apoptosis Detection kit (BD, USA). Briefly, trypsinized cell pellets were washed twice with cold PBS and then resuspend in $1 \mathrm{X}$ Binding Buffer at a concentration of $1 \times 10^{6}$ cells $/ \mathrm{ml}$. Then, $100 \mu 1$ of the solution was transferred to a culture tube, adding $5 \mu 1$ of FITC-Annexin $\mathrm{V}$ and $5 \mu 1$ propidium iodide for incubating for $15 \mathrm{~min}$. Cells were flow-sorted, and analyzed using a FACS machine (Becton-Dickinson, Mountain View, CA). All FACS analyses were performed at least 3 times in duplicate samples and yielded reproducible results.

Cell adhesion assay. Cell adhesion assay was performed essentially as described (18). In brief, 96-well, flat-bottom culture plates were coated with $50 \mu 1$ of Matrigel (BD, USA) in serum-free DMEM medium overnight at $4^{\circ} \mathrm{C}$. The cells after RNAi treatment for $48 \mathrm{~h}$ of control, Mock group and RNAi group were harvested with trypsin/EDTA, washed 


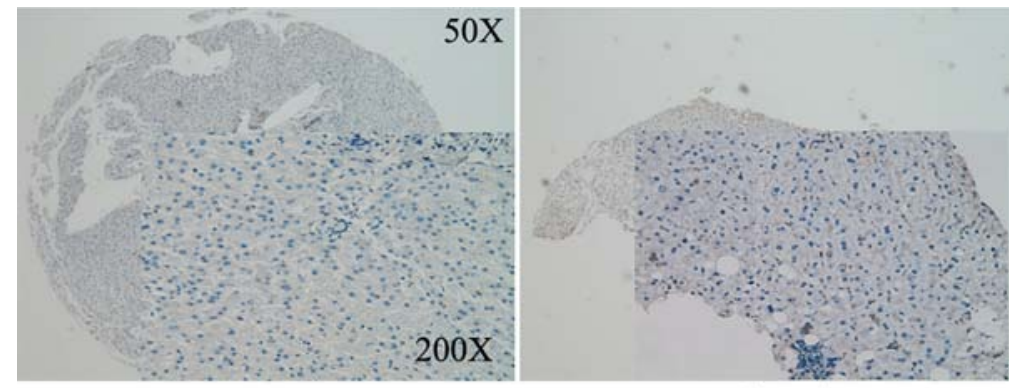

normal

hepatic

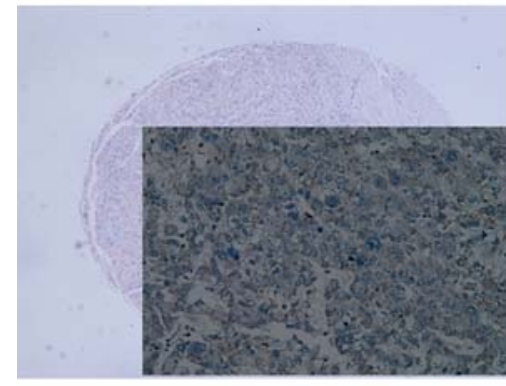

cirrhosis

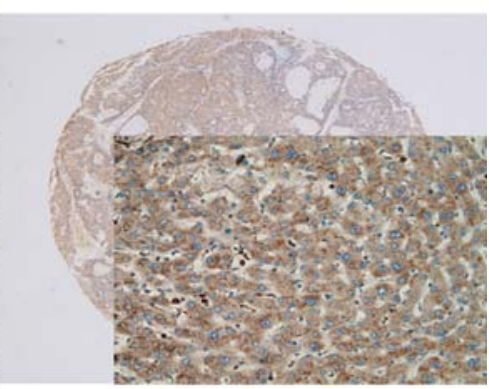

$\mathrm{HCC}$

Figure 1. Stathmin expression was up-regulated in HCC tissues. TMA of normal liver, hepatitis, hepatic cirrhosis and HCC tissues showed that stathmin was overexpressed in HCC, compared with HBV and cirrhosis tissues, while it could not detect in normal liver tissue.

twice and resuspended in DMEM medium. Cells $\left(2.5 \times 10^{4} /\right.$ well) were added to each well in triplicate and incubated for $20 \mathrm{~min}, 40 \mathrm{~min}$ and $60 \mathrm{~min}$ at $37^{\circ} \mathrm{C}$. Plates were then washed three times with PBS to remove unbound cells. The cells remaining attached to the plates were counted (5 fields/well).

In vitro invasion and migration assay. In vitro invasion assay was performed using 24-well Transwell unit with polycarbonate filters (Corning Costar, Cambridge, MA). The HCCLM3 cells with RNAi treatments were placed in the upper part of the Transwell, Mock group and control group, were incubated for indicated time, fixed with $4 \%$ paraformaldehyde and stained with $10 \%$ Giemas for $10 \mathrm{~min}$. Cells in the upper chamber were removed by cotton swab and the cells that invaded through the Matrigel and were located on the underside of the filter ( 5 fields/filter) were counted. Three invasion chambers were used per condition. The values obtained were calculated by averaging the total number of cells from three filters. Experimental procedures of in vitro migration assay are the same as the in vitro invasion assay described above except that the filter was not coated with Matrigel for the migration assay.

Statistical analysis. All data are expressed as the mean \pm SD for 3 independent experiments using three difference preparations. The difference between the means was assessed by Student's t-test and was considered as statistically significant when the P-value was $<0.05$.

\section{Results}

Expression of stathmin during hepatocarcinogenesis. The expressions of stathmin in liver tissue samples from healthy individuals $(n=24)$, individuals with hepatitis $B(n=24)$, individuals with cirrhosis $(n=24)$ and individuals with HCC $(n=24)$ were detected by IHC in TMAs. The results showed that
Table I. Correlation between stathmin expression and the different tissues during hepatocarcinogenesis.

\begin{tabular}{lcc}
\hline & \multicolumn{2}{c}{ Stathmin expression } \\
\cline { 2 - 3 } $\begin{array}{l}\text { Different } \\
\text { tissues }\end{array}$ & $\begin{array}{c}\text { Low and nil } \\
\text { expression }\end{array}$ & $\begin{array}{c}\text { High } \\
\text { expression }\end{array}$ \\
\hline Normal liver & 23 & 1 \\
HBV & 21 & $3^{\mathrm{a}, \mathrm{c}}$ \\
Cirrhosis & 21 & $3^{\mathrm{a}, \mathrm{c}}$ \\
Non-metastatic HCC & 18 & $54^{\mathrm{b}, \mathrm{c}}$ \\
Metastatic HCC & 5 & $43^{\mathrm{b}, \mathrm{d}}$ \\
\hline
\end{tabular}

Stathmin expression was correlated with the different tissues during hepatocarcinogenesis. Intensity of staining was scored as $0(-), 1(+)$, $2(++)$ or $3(+++)$, and the extent of staining was based on the percentage of positive tumor cells: $0(<5 \%), 1(5-25 \%), 2(26-50 \%)$ and $3(>50 \%)$. The final score of each sample was assessed by summarizing the result of intensity and extent of staining. Zero grade was low and nil expression, 1-3 grade was high expression. ${ }^{\text {aStatistical }}$ analyses were conducted with Fisher's exact test for all the parameters. ${ }^{\text {aP-values }}<0.05$ and ${ }^{\mathrm{b}} \mathrm{P}$-values $<0.01$ were considered statistically significant. ${ }^{~}$ The test group compared with normal liver tissues group; dhe detective group compared with non-metastatic HCC tissues group.

stathmin expression was up-regulated in $\mathrm{HCC}$, compared with normal liver, hepatitis and cirrhosis samples $(\mathrm{P}<0.01)$. It could hardly be detected in normal liver tissues and no significant difference of stathmin expression was observed between hepatitis and cirrhosis samples, but the stathmin staining level was slightly but significant higher in hepatitis and cirrhosis tissue as compared to normal tissue $(\mathrm{P}<0.05)$ (Fig. 1, Table I). Moreover, stathmin expression was further tested in TMA 


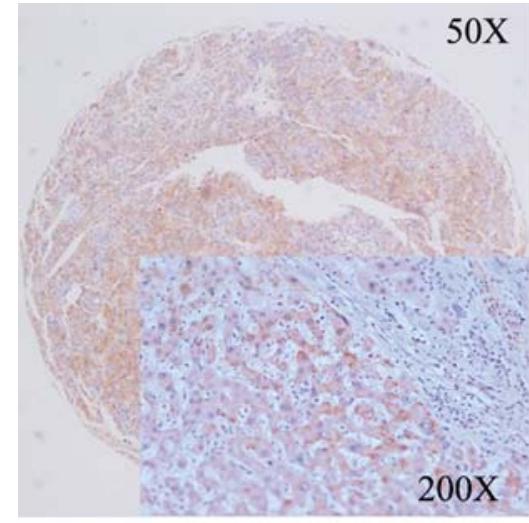

non-metastatic HCC

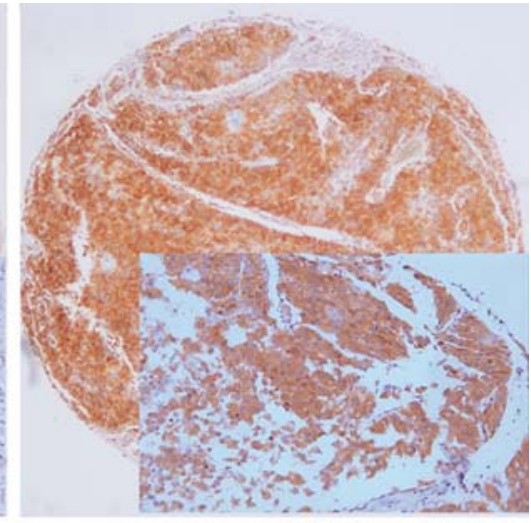

metastatic $\mathrm{HCC}$

Figure 2. Stathmin expression was up-regulated in metastatic HCC tissues. The expression of stathmin was clearly up-regulated in metastatic HCC specimens compared with primary HCC in TMA.

A
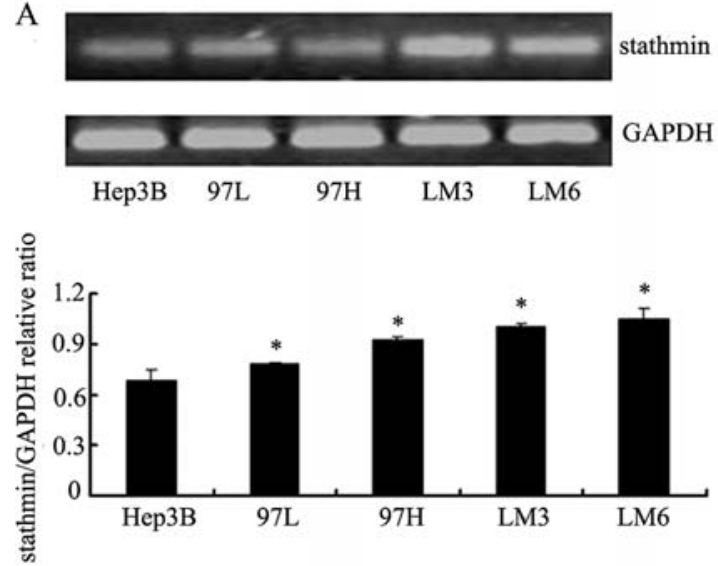

B
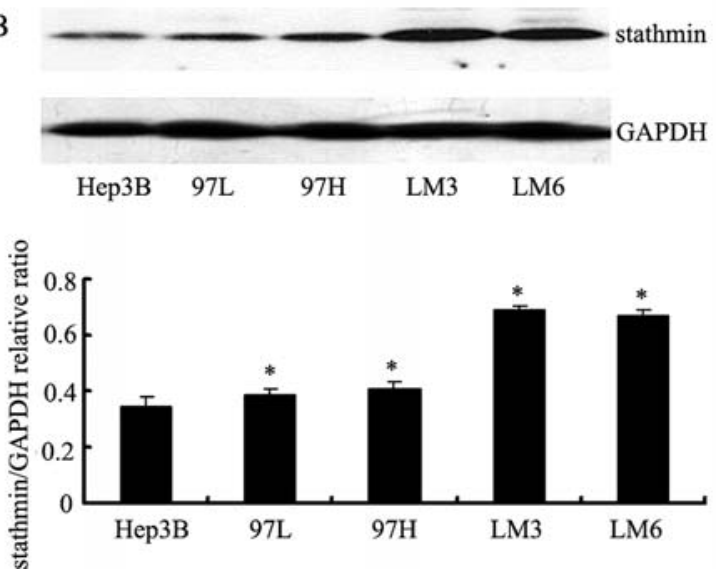

Figure 3. Stathmin expression stepwise induced in HCC cell lines with metastatic potential. (A) Stathmin mRNA expression was stepwise up-regulated in HCC cell lines with different metastatic potential, RT-PCR analysis of GAPDH expression was used as normalization control. (B) Western blot analysis of stathmin expression in HCC cell lines with metastatic potential is shown, GAPDH expression was used as normalization control. Statistical analysis of the stathmin/GAPDH ratio in different HCC cell lines were used and evaluated by densitometric scanning of the results. The data from three independent experiments are presented as rations of the control levels and as are shown as mean $\pm \mathrm{SEM} .{ }^{*} \mathrm{P}<0.05$.

which included 48 non-metastatic HCC tissue dots and 48 metastatic HCC tissue dots. It showed that stathmin expression was significantly increased in metastatic HCC specimens, compared with primary HCC $(\mathrm{P}<0.01)$ (Fig. 2, Table I). All these findings suggested consecutive up-regulation of stathmin expression was associated with hepatocarcinogenesis.

In addition, stathmin expression in a series of HCC cell lines which have similar genetic background but with gradually up-regulated metastatic potential was measured by RT-PCR and Western blot analysis. It was observed that mRNA and protein levels of stathmin were highest in HCCLM3 and HCCLM6 cells with high metastatic potential, and the lowest in Hep3B cells (Fig. 3). It indicated that upregulated expression of stathmin was relative to the HCC cell metastatic ability.

Knockdown of stathmin in HCCLM3 cells by small interfering RNA (siRNA)-mediated transfection. In the present study, siRNA mediated-transfection was used to determine effects of stathmin expression silencing on metastatic HCC cell behavior. To optimize conditions for siRNA knockdown, we first determined transfection efficiency using FITC Fluorescent control. Twenty-four hours after transfection, HCCLM3 cells were observed using a fluorescent microscope. The transfection efficiency was evaluated by counting the green fluorescent cells, which was over $95 \%$ when using $80 \mathrm{nM} / \mathrm{l}$ final concentration of siRNA sand $1 \times 10^{5} / \mathrm{ml}$ HCCLM3 cells. Therefore, we used $80 \mathrm{nM} / 1$ siRNAs and $1 \times 10^{5} / \mathrm{ml}$ HCCLM3 cells to perform subsequent experiments.

After transfection of HCCLM3 cells with three different siRNAs targeting the stathmin gene, mRNA and protein levels of stathmin was detected by RT-PCR and Western blot at $48 \mathrm{~h}$ after transfection. The results showed decrease of stathmin mRNA and protein levels with the siRNAs while the levels of the house-keeping gene GAPDH remained relatively unchanged. Relative quantification analysis revealed 


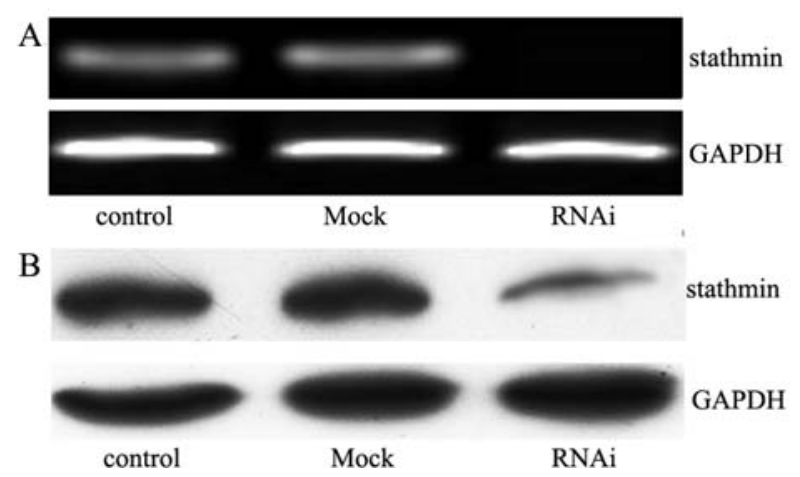

Figure 4. Effect of stathmin knockdown on HCCLM3 is shown. (A) Stathmin mRNA level was decreased in HCCLM3 cells as examined by RT-PCR after siRNA-mediate transfection. (B) Stathmin protein level was decreased as shown by Western blotting after $48 \mathrm{~h}$ transfection.

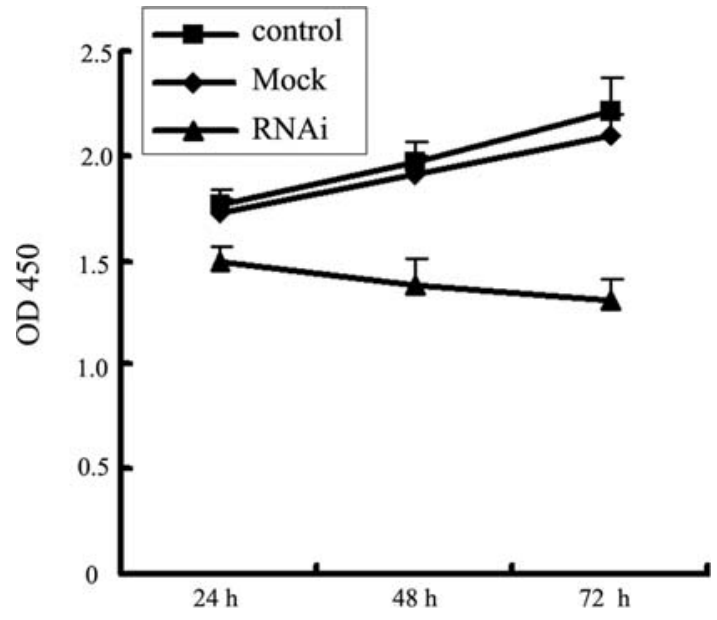

Figure 5. HCCLM3 cell proliferation was inhibited by stathmin knockdown. Cell viability was evaluated by using the CCK8 assay after stathmin silencing The data from three independent experiments are presented and shown as mean \pm SEM. ${ }^{*} \mathrm{P}<0.05$.

that siRNA-1 was more effective in silencing stathmin expression than siRNA-2 and siRNA-3 (Fig. 4). Altogether, HCCLM3 cells were transfected with siRNA-1 (RNAi) or control non-specific siRNA (Mock) and non-treated HCCLM3 cells (control) were chosen for further study.
Inhibition of HCCLM3 cell proliferation by stathmin expression silencing in vitro. CCK8 analysis was performed to test the effect of siRNA-mediated stathmin expression knockdown on proliferation of HCCLM3 cells. HCCLM3 cells were transfected with siRNA-1 against stathmin gene and were either maintained in the presence or absence of $80 \mathrm{nM}$ siRNA for 24, 48 and $72 \mathrm{~h}$. Untransfected HCCLM3 cells were grown under the same conditions and used as a control. The cell proliferation was decreased significantly after treatment with siRNA-1 at 24, 48 and $72 \mathrm{~h}$ as compared with the control (Fig. 5, $\mathrm{P}<0.05$ ). The inhibition rates of cell proliferation were 13.0, 28.0 and $37.3 \%$ after 24,48 and $72 \mathrm{~h}$ of transfection, respectively. In contrast, transfection of the negative control had no obvious effect on cell proliferation.

Increasing of HCCLM3 apoptosis by knockdown of stathmin expression. To determine the effect of knockdown stathmin expression on HCCLM3 cell apoptosis, flow cytometry was used to analyze cell apoptosis through FITC-Annexin V and PI labeling. It showed that the percentage of apoptotic cells was increased to $26.6 \%$ in RNAi group, compared with $9.1 \%$ in Mock group (Fig. 6). In addition, stathmin knockdown induced cell cycle arrest by inhibiting progression from G2 to $\mathrm{S}$ phase. By single-cell analysis using FACS, our results showed that there was no significant difference in HCCLM3 cell cycle before and after stathmin knockdown by RNAi.

Inhibition of HCCLM3 adhesion, migration and invasion in vitro by siRNA-mediated stathmin knockdown. To determine whether stathmin plays a crucial role in adhesion and motility, we firstly demonstrated the effect of stathmin knockdown in HCCLM3 cells on cell-ECM contact. The result showed that number of the RNAi group cell adhesion to Matrigel $(29 \pm 2.7 ; 36.7 \pm 5.5 ; 49.7 \pm 7.6)$ was markedly less than Mock group cells $(50.6 \pm 4 ; 6.7 \pm 5 ; 102.3 \pm 6)$ and control group cells $(49.1 \pm 6.6 ; 66.8 \pm 3.7 ; 105.1 \pm 9.7)$ at the time-point of 20,40 and 60 min, respectively $(\mathrm{P}<0.05)$ (Fig. 7A).

Migration and invasion assay in vitro were carried out to evaluate stathmin knockdown on HCCLM3 cell motility. The result showed that the average number of invaded and migrated HCCLM3 cells transfected with siRNA significantly decreased in comparison with the Mock group and control group cells $(\mathrm{P}<0.05)$, reduced by $42.6 \pm 3.3 \%$ and $60.1 \pm 0.8 \%$ compared with Mock group, respectively (Fig. 7B).

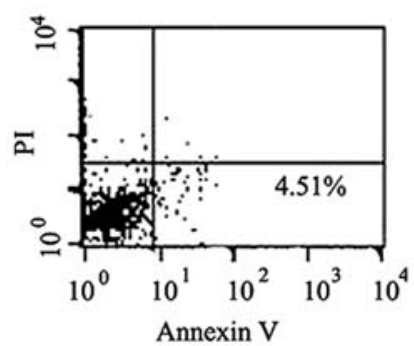

control
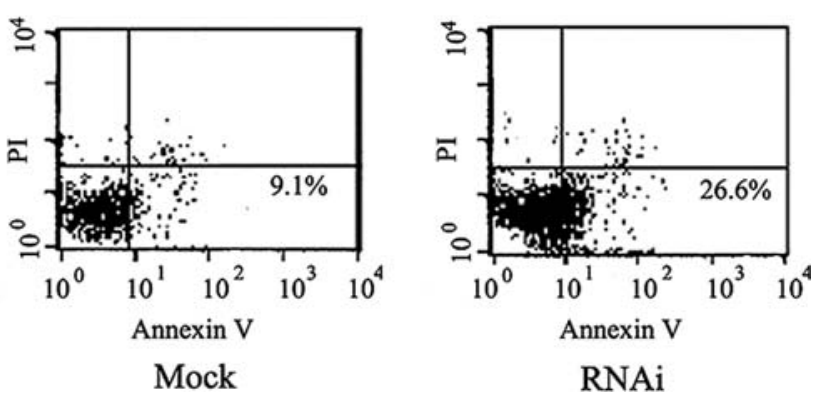

Figure 6. HCCLM3 apoptosis was induced by silencing of stathmin. Stathmin knockdown induced apoptosis in the RNAi group in HCCLM3 cells. After RNA interference, the cells were stained with Annexin V (x-axis) and propidium iodide (y-axis) to quantify apoptosis and necrosis, respectively. The illustrated flow cytometry histograms are representative of three separate experiments. 
A

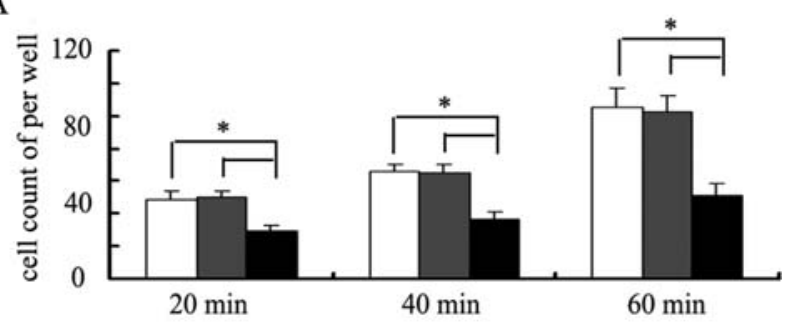

B

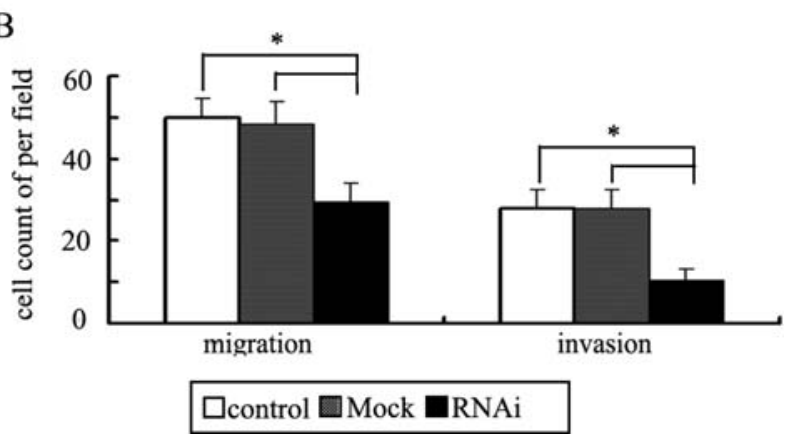

Figure 7. Effect of stathmin knockdown on adhesion, motility and migration of HCCLM3. (A) The cells that adhered with Matrigel-coated 96-well plate were counted as total cell number per well after RNAi treatment for $48 \mathrm{~h}$ of control group, Mock group and RNAi group. (B) HCCLM3 was transfected with control, non-targeting siRNA and stathmin siRNA for $48 \mathrm{~h}$, replated onto uncoated or Matrigel-coated transwell chambers and incubated for $48 \mathrm{~h}$. The cells that penetrated to the opposite side of the membrane were stained with Giemsa, and those in four randomly chosen fields were counted. The panels depict the mean \pm SEM of the migration and invasion ratios, determined in three independent experiments. ${ }^{*} \mathrm{P}<0.05$ vs. Mock group.

\section{Discussion}

Hepatocarcinogenesis is believed to be a multistep process in which a series of molecular alterations is accumulating. Stathmin, a depolymerizer of microtubules, plays an important role in cell mitosis and motility. Dysfunction of stathmin could lead to constant microtubule assembly and uncontrolled cell cycles, which result in continuous abnormal cell growth tumor formation. However, it is unclear whether stathmin contributes to hepatocarcinogenesis or not. In this study, we detected the expression of stathmin in various liver tissues associated with hepatocarcinogenesis and HCC cell lines with different metastatic potentials, and investigated the role of stathmin in HCC cell proliferation, apoptosis, adhesion, migration and invasion.

It is known that stathmin is expressed at high levels in a wide variety of human malignancies, and high levels of stathmin expression in cancer cells were shown to correlate with their proliferative potential and seem to be necessary for the maintenance of their malignant phenotype. Hence, it was proposed that the level of expression of stathmin may serve as a biomarker in some cancers. Our data from histological and cytological detection suggested that stathmin expression was up-regulated with the progression of hepatocarcinogenesis. It is reported that stathmin is abundantly expressed in fetal liver, on the contrary, dramatically decreased in adult liver (19). In our study, stathmin expression was negative in normal liver tissue, low in hepatitis and hepatic cirrhosis tissues and significantly up-regulated in HCC tissue. It suggests that some pathogens including inflammatory factors could induce stathmin expression in liver tissue. Furthermore, up-regulated expression of stathmin was also relative to HCC metastasis, consistent with some literature (20-23). Interestingly, stathmin expression is similar to alpha fetoprotein (AFP), an important biomarker for $\mathrm{HCC}$ diagnosis and prognosis. All these findings imply that stathmin may be an important molecular associated with hepatocarcinogenesis and HCC metastasis.

It is reported that there is a close link between stathmin expression and regulation of cellular proliferation in leukocytes, undifferentiated multi-potential embryonal carcinoma cells, breast cancer and ovarian cancer cells. It is also proven that stathmin may play a role in the $\mathrm{p} 34^{\mathrm{cdc} 2}$-regulated pathway, which controls entry into mitosis and proliferation $(24,25)$. In our study, we achieved effective down-regulation of stathmin expression by using siRNA treatment strategy in HCC cell lines. Experimental data showed that transient stathmin downregulation led to significant inhibition of in vitro proliferation, accumulation of $\mathrm{G} 2 / \mathrm{M}$ phase, and apoptosis of $\mathrm{HCC}$ cells. However, accumulation of G2/M phase through knockdown of stathmin was not obvious, although it is reported that both overexpression and inhibition of expression of stathmin results in mitotic arrest, which may be attributable to genetic characteristics of our HCC cell lines.

In addition, our results demonstrated that stathmin is able to promote HCC cell adhesion, migration and invasion in vitro. It is reported that reducing the expression of stathmin inhibited the migration of neurons in vivo in mice and Drosophila, while in germ and border cells had the same effect (26-28). It was proven that embryonic fibroblast cells from stathmindeficient mice migrated slower than those from wild-type animals, and the phenotype can be changed by up-regulating stathmin expression. Another clarifying example is given by the group of Baldassarre that cytosolic p27 kip, a cyclindependent kinase inhibitor, inhibit ECM-driven cell migration in the fibrosarcoma HT-1080 cells, by interacting with stathmin and inactivating the microtubule-depolymerization activity of stathmin. Moreover, stathmin MTdepolymerizing activity is negatively regulated by phosphorylation (29). These findings indicate that stathmin regulates cell motility or adhesion through complicated mechanisms, further research need to be carried out to elucidate how stathmin is involved in HCC metastasis.

In conclusion, our findings indicate that stathmin may be a crucial factor during hepatocarcinogenesis and contributes to HCC cell proliferation, apoptosis and motility by RNA interference. Therefore, stathmin appears to be a promising therapeutic target of HCC using a RNAi-based approach alone or in combination with chemotherapeutics. However, further studies are needed to investigate the mechanism how stathmin is involved in HCC cells proliferation and motility.

\section{Acknowledgements}

This study was financially supported by China National HighTech Research and Development Program (2006AA02A308), China National Key Projects for Infectious Disease (2008ZX 10002-021 and 2008ZX10002-017). 


\section{References}

1. Parkin DM, Bray F, Ferlay J and Pisani P: Global cancer statistics, 2002. CA Cancer J Clin 55: 74-108, 2005.

2. El-Serag HB and Rudolph KL: Hepatocellular carcinoma: epidemiology and molecular carcinogenesis. Gastroenterology 132: 2557-2576, 2007.

3. Colombo M: Hepatocellular carcinoma. J Hepatol 15: 225-236, 1992.

4. Sun M, Eshleman JR, Ferrell LD, Jacobs G, Sudilovsky EC Tuthill R, Hussein MR and Sudilovsky O: An early lesion in hepatic carcinogenesis: loss of heterozygosity in human cirrhotic livers and dysplastic nodules at the 1p36-p34 region. Hepatology 33: 1415-1424, 2001

5. Boige V, Laurent-Puig P, Fouchet P, Flejou JF, Monges G, Bedossa P, Bioulac-Sage P, Capron F, Schmitz A, Olschwang S and Thomas G: Concerted non-syntenic allelic losses in hyperploid hepatocellular carcinoma as determined by a highresolution allelotype. Cancer Res 57: 1986-1990, 1997.

6. Kusano N, Shiraishi K, Kubo K, Oga A, Okita K and Sasaki K: Genetic aberrations detected by comparative genomic hybridization in hepatocellular carcinomas: their relationship to clinico-pathological features. Hepatology 29: 1858-1862, 1999.

7. Piao Z, Kim H, Malkhosyan S and Park C: Frequent chromosomal instability but no microsatellite instability in hepatocellular carcinomas. Int J Oncol 17: 507-512, 2000.

8. Katoh H, Shibata T, Kokubu A, Ojima H, Loukopoulos P, Kanai Y, Kosuge T, Fukayama M, Kondo T, Sakamoto M, Hosoda F, Ohki M, Imoto I, Inazawa J and Hirohashi S: Genetic profile of hepatocellular carcinoma revealed by array-based comparative genomic hybridization: identification of genetic indicators to predict patient outcome. J Hepatol 43: 863-874, 2005.

9. Fang W, Piao Z, Simon D, Sheu JC and Huang S: Mapping of a minimal deleted region in human hepatocellular carcinoma to 1p36.13-p36.23 and mutational analysis of the riz (prdm2) gene localized to the region. Genes Chromosomes Cancer 28: 269-275, 2000.

10. Leung TH, Wong N, Lai PB, Chan A, To KF, Liew CT, Lau WY and Johnson PJ: Identification of four distinct regions of allelic imbalances on chromosome 1 by the combined comparative genomic hybridization and microsatellite analysis on hepatocellular carcinoma. Mod Pathol 15: 1213-1220, 2002.

11. Koshikawa K, Nomoto S, Yamashita K, Ishigure K, Takeda S and Nakao A: Allelic imbalance at 1p36 in the pathogenesis of human hepatocellular carcinoma. Hepatogastroenterology 51: 186-191, 2004

12. Nishimura T, Nishida N, Itoh T, Komeda T, Fukuda Y, Ikai I, Yamaoka Y and Nakao K: Discrete breakpoint mapping and shortest region of overlap of chromosome arm 1q gain and $1 \mathrm{p}$ loss in human hepatocellular carcinoma detected by semiquantitative microsatellite analysis. Genes Chromosomes Cancer 42: 34-43, 2005.

13. Ozon S, Byk T and Sobel A: Sclip: a novel scg10-like protein of the stathmin family expressed in the nervous system. J Neurochem 70: 2386-2396, 1998.

14. Pellier-Monnin V, Astic L, Bichet S, Riederer BM and Grenningloh G: Expression of scg10 and stathmin proteins in the rat olfactory system during development and axonal regeneration. J Comp Neurol 433: 239-254, 2001.
15. Curmi PA, Nogues C, Lachkar S, Carelle N, Gonthier MP, Sobel A, Lidereau R and Bieche I: Overexpression of stathmin in breast carcinomas points out to highly proliferative tumours. Br J Cancer 82: 142-150, 2000.

16. Price DK, Ball JR, Bahrani-Mostafavi Z, Vachris JC, Kaufman JS, Naumann RW, Higgins RV and Hall JB: The phosphoprotein op18/stathmin is differentially expressed in ovarian cancer. Cancer Invest 18: 722-730, 2000.

17. Gao Q, Qiu SJ, Fan J, Zhou J, Wang XY, Xiao YS, Xu Y, Li YW and Tang $\mathrm{ZY}$ : Intratumoral balance of regulatory and cytotoxic $t$ cells is associated with prognosis of hepatocellular carcinoma after resection. J Clin Oncol 25: 2586-2593, 2007.

18. Spessotto P, Giacomello E and Perri R: Improving fluorescencebased assays for the in vitro analysis of cell adhesion and migration. Mol Biotechnol 20: 285-304, 2002.

19. Bieche I, Maucuer A, Laurendeau I, Lachkar S, Spano AJ, Frankfurter A, Levy P, Manceau V, Sobel A, Vidaud M and Curmi PA: Expression of stathmin family genes in human tissues: non-neural-restricted expression for sclip. Genomics 81: 400-410, 2003.

20. Ghosh R, Gu G, Tillman E, Yuan J, Wang Y, Fazli L, Rennie PS and Kasper S: Increased expression and differential phosphorylation of stathmin may promote prostate cancer progression. Prostate 67: 1038-1052, 2007.

21. Belletti B, Nicoloso MS, Schiappacassi M, Berton S, Lovat F, Wolf K, Canzonieri V, D'Andrea S, Zucchetto A, Friedl P, Colombatti A and Baldassarre G: Stathmin activity influences sarcoma cell shape, motility and metastatic potential. Mol Biol Cell 19: 2003-2013, 2008.

22. Brattsand G: Correlation of oncoprotein 18/stathmin expression in human breast cancer with established prognostic factors. Br J Cancer 83: 311-318, 2000

23. Xi W, Rui W, Fang L, Ke D, Ping G and Hui-Zhong Z: Expression of stathmin/op18 as a significant prognostic factor for cervical carcinoma patients. J Cancer Res Clin Oncol 135: 837-846, 2009.

24. Luo XN, Mookerjee B, Ferrari A, Mistry S and Atweh GF: Regulation of phosphoprotein p18 in leukemic cells. Cell cycle regulated phosphorylation by p34cdc2 kinase. J Biol Chem 269: 10312-10318, 1994.

25. Brattsand G, Marklund U, Nylander K, Roos G and Gullberg M: Cell-cycle-regulated phosphorylation of oncoprotein 18 on ser16, ser25 and ser38. Eur J Biochem 220: 359-368, 1994.

26. Borghese L, Fletcher G, Mathieu J, Atzberger A, Eades WC, Cagan RL and Rorth P: Systematic analysis of the transcriptional switch inducing migration of border cells. Dev Cell 10: 497-508, 2006.

27. Jin K, Mao XO, Cottrell B, Schilling B, Xie L, Row RH, Sun Y, Peel A, Childs J, Gendeh G, Gibson BW and Greenberg DA: Proteomic and immunochemical characterization of a role for stathmin in adult neurogenesis. FASEB J 18: 287-299, 2004.

28 Ozon S, Guichet A, Gavet O, Roth S and Sobel A: Drosophila stathmin: a microtubule-destabilizing factor involved in nervous system formation. Mol Biol Cell 13: 698-710, 2002.

29. Larsson N, Marklund U, Gradin HM, Brattsand G and Gullberg M: Control of microtubule dynamics by oncoprotein 18: dissection of the regulatory role of multisite phosphorylation during mitosis. Mol Cell Biol 17: 5530-5539, 1997. 